\title{
Alterações fisiológicas ocasionadas pela COVID-19 e suas consequências no agravamento da doença: graduandos de farmácia aplicando o aprendizado de bioquímica clínica
}

\author{
Physiological changes caused by COVID-19 and its consequences in the \\ worsening of the disease: Pharmacy graduates applying Clinical Biochemistry \\ learning
}

Cláudia Rebouças da Silva ${ }^{1}$; Ediel da Silva Barreto ${ }^{1}$; Larissa de Jesus Santana ${ }^{1}$; Layse Ribeiro Borges $^{1}$; Yuri Ribeiro da Silva ${ }^{1}$; Rita Terezinha de Oliveira Carneiro ${ }^{2 *}$

\begin{abstract}
${ }^{1}$ Graduação em andamento em Farmácia. Faculdade Maria Milza - FAMAM, Governador Mangabeira - Bahia, Brasil, 44350-000, claudreboucas@gmail.com; https://orcid.org/0000-0002-0326092X; ediel_barreto@hotmail.com;https://orcid.org/0000-0002-8684-572X;
\end{abstract} https://orcid.org/0000-0002-6273-2122; laysenayelle@ hotmail.com; https://orcid.org/0000-0002-5107-0995; yuriribeiro1898@gmail.com; https://orcid.org/0000-0002-9731-4270;

${ }^{2 *}$ Docente do curso de Farmácia. Faculdade Maria Milza - FAMAM, Governador Mangabeira - Bahia, Brasil, 44350-000, ritaterezinha@gmail.com; https://orcid.org/0000-0002-7568-6487

\section{Resumo}

COVID-19 é uma infecção altamente letal descrita recentemente na história da humanidade. Suas manifestações clínicas são variáveis, mas passíveis de serem analisadas por testes laboratoriais já amplamente empregados. Farmacêuticos são importantes atores na orientação de pacientes quanto aos cuidados que devem ser tomados durante o curso da pandemia. $\mathrm{O}$ objetivo foi promover a discussão entre graduandos de Farmácia de uma faculdade do Recôncavo Baiano sobre as principais alterações fisiológicas ocorridas em virtude da COVID19 e suas consequências na evolução clínica dos infectados. Trata-se de uma metanálise, cuja metodologia consistiu em busca de trabalhos completos publicados em bases de dados (PubMed, Scielo e Google Acadêmico). Foram analisados 33 artigos dos quais foram extraídas informações sobre 5 parâmetros: liberação de mediadores inflamatórios; mudança no 
metabolismo de carboidratos e as alterações nas atividades cardíaca, renal e pancreática considerados como preditores de agravamento nos casos de COVID-19. O trabalho apresenta limitações sobre tempo para sua execução e devido falhas na conexão durante as interações on line com os estudantes. A perspectiva é que os participantes desse estudo possam contribuir com esclarecimento sobre os possíveis agravos na saúde causados pela COVID-19 aos usuários das instituições nas quais eles atuam como estagiários.

Palavras-chave: Atenção Farmacêutica; pandemia; uso irracional de fármacos.

\begin{abstract}
COVID-19 is a highly lethal infection limited recently in human history. Its clinical manifestations are variable, but can be analyzed by laboratory tests that are already widely used. Pharmacists are important players in guiding patients on the care that should be taken during the course of the pandemic. The objective was to promote the discussion among Pharmacy graduates from a college in Reconcavo Baiano about the main physiological changes that occurred due to COVID-19 and its consequences on the clinical evolution of those infected. This is a meta-analysis, the methodology consisted of searching for works published in databases (PubMed, Scielo and Google Scholar). 33 articles were released from which information was extracted on 5 parameters: release of inflammatory mediators; change without carbohydrate metabolism and changes in cardiac, renal and pancreatic activities considered as predictors of worsening in cases of COVID-19. The work has limitations on the time for its execution and due to flaws in the connection during interactions on the line with students. The perspective is that the participants of this study contribute with clarification about the possible health problems caused by COVID-19 to users of the institutions in which they work as interns.
\end{abstract}

Keywords: Irrational use of drugs; pandemic; Pharmaceutical Care.

\title{
1. Introdução
}

A COVID-19 (Corona Virus Disease 2019) é o mais urgente problema de saúde pública da atualidade com notificação mundial de 83.715.617 casos confirmados da doença, e apresenta alta letalidade devido ao registro de 1.835.901 óbitos em sua decorrência, até o presente momento (WHO, 2020). As características clínicas da COVID-19 apresentam um espectro amplo, haja vista que a sintomatologia pode variar desde uma síndrome gripal a um quadro de insuficiência respiratória aguda; além dos casos assintomáticos (XAVIER et al., 2020; WU et al., 2020).

O primeiro relato da infecção ocorreu em dezembro de 2019. Em virtude da sua recente descrição ainda existem lacunas no entendimento quanto a virulência do novo coronavírus 
identificado como SARS-coV-2 (Severe Acute Respiratory Syndrome coronavírus 2), e na elucidação sobre os mecanismos de defesa imunológica desenvolvidos pelos indivíduos infectados (HARAPAN et al., 2020). Em concomitância ao momento em que países como Inglaterra e Estados Unidos conduzem a terceira fase dos testes com vacina contra a COVID19 (CALLAWAY, 2020), também são registrados os primeiros casos de reinfecção pelo SARScov-2 no Brasil (BRASIL, 2020).

O diagnóstico da COVID-19 é realizado por meio de testes laboratorial (dosagem de anticorpos totais) e de biologia molecular (reverse-transcriptase polymerase chain reaction, qPCR), além dos exames de bioimagem (radiografia e tomografia computadorizada). A análise clínica-laboratorial se configura como ferramenta imprescindível na caracterização da doença, revelando a evolução quanto a cronicidade ou resolução dos casos (LI et al., 2020a). Os resultados bioquímicos são também empregados na elucidação dos protocolos terapêuticos destinados aos pacientes em estado grave da doença e na caracterização epidemiológica da doença (LIMA et al., 2020; SOUZA et al., 2020).

Atenção Farmacêutica é caracterizada como uma prestação de serviço realizada por farmacêuticos que consiste na interação entre esse profissional e os usuários da farmácia com intuito de promover a dispensação adequada dos fármacos, orientar sobre a farmacoterapia e sobre a adesão do paciente ao seu respectivo tratamento (SILVA \& ARAUJO, 2020). Portanto, farmacêuticos também atuam na linha de frente no combate a COVID-19, no sentido de disseminar informações verdadeiras sobre a doença (CAGNAZZO \& CHIARI-ANDRÉO, 2020), especialmente orientando sobre a possibilidade de ocorrer efeitos adversos devido ao uso irracional de antibióticos, glicocorticoides, antipiréticos, analgésicos e anti-inflamatórios que em diferentes momentos do curso da pandemia foram erroneamente sugeridos como perspectiva de terapia e prevenção da COVID-19 (SILVA \& ARAUJO, 2020; TRITANY \& TRITANY, 2020). Contudo, sem qualquer eficácia comprovada (prescrição off label) (BRASIL, 2012).

O objetivo desse trabalho foi discutir sobre as principais alterações fisiológicas no ser humano ocorridas em virtude da COVID-19 e suas consequências na evolução clínica dos infectados com enfoque para os casos graves da doença, a partir da visão dos alunos de 
graduação do $4^{\mathrm{o}}$ semestre do curso de bacharelado em Farmácia de uma Faculdade do Recôncavo da Bahia, Brasil. Considerando a urgência na caracterização da doença e na adoção de medidas preventivas quanto a ocorrência de novos casos, a proposta desse trabalho é justificável por contribuir com a sensibilização dos farmacêuticos em formação sobre mecanismos fisiológicos desencadeados na infecção pelo SARS-coV-2, com vistas a melhorar a prestação de serviço que esses profissionais poderão futuramente oferecer no âmbito da Atenção Farmacêutica.

\section{Material e Métodos}

Esse trabalho foi proposto inicialmente como uma atividade avaliativa referente a disciplina Bioquímica Clínica, ofertada no semestre de 2020.2 aos graduandos do $4^{\circ}$ semestre de Farmácia da Faculdade Maria Milza (FAMAM). Trata-se de uma metanálise sobre publicações cientificas na temática de biomarcadores utilizados na análise laboratorial das principais alterações fisiológicas desencadeadas pela COVID-19.

A busca pelos artigos foi realizada em bases de dados: PubMed, Scielo e Google Acadêmico usando os descritores: "alterações fisiológicas da COVID-19"; "sintomas da COVID-19"; "diagnóstico laboratorial da COVID-19" nos idiomas português e inglês. Os critérios de inclusão adotados na seleção dos artigos foram: $i$ ) trabalhos completos; ii) qualis (A1 a B2) e/ou fator do impacto (>2) dos periódicos. E os critérios de exclusão: $i$ ) material de divulgação elaborado por indústrias farmacêuticas responsáveis pela produção ou distribuição de fármacos empregados nos protocolos terapêuticos estabelecidos durante no curso da infecção; ii) publicações do tipo carta ao editor, casos clínicos e informes técnicos.

A análise dos dados foi conduzida pelos seis autores/pesquisadores envolvidos na pesquisa seguindo os critérios adotados por Minayo (2001), que consiste em três etapas: análise prévia do conteúdo apresentados pelos autores (organização dos dados); exploração do conteúdo de cada artigo (leitura e confronto dos dados apresentados pelos autores) e tratamento dos dados (interpretação das informações obtidas).

\section{Resultados}


Foram encontrados 6.859 publicações nas bases de dados consultadas. Todavia, pela adequação aos critérios de inclusão e exclusão adotados nesse trabalho 100 artigos $(2,4 \%$, $\mathrm{N}=7.000$ ) foram considerados. Uma segunda análise foi realizada com os 100 trabalhos selecionados na etapa anterior, dentre os quais foram excluídos: 25 Cartas ao editor; 18 Estudos de casos; 17 Informes técnicos e 7 trabalhos em duplicidade. A Figura 1 ilustra as etapas de seleção dos trabalhos

Figura 1. Fluxograma ilustrando as etapas de obtenção, seleção e análise dos textos analisados nesse trabalho.

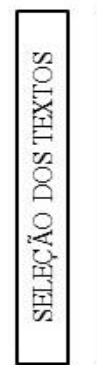

Aplicação dos critérios

de inclusão e

exclusão:

$N=100$ artigos

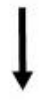

Eliminação de cartas ao editor; informe técnico e casos clínicos

$N=33$ artigos

PubMed $=23$

Scielo $=3$

Google Acadêmico $=7$

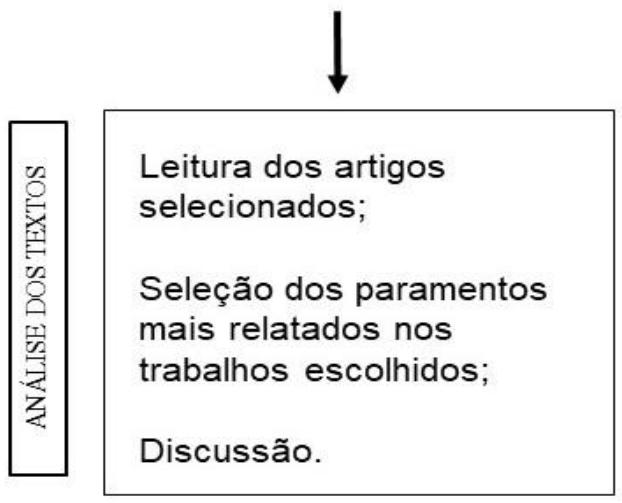


Dentre as diversas alterações clínicas causadas pela COVID-19, para essa análise foram consideradas aquelas citadas em, pelo menos três dos 33 trabalhos analisados, as quais estão sumarizadas na Tabela 1 e serão discutidas a seguir.

Tabela 1. Principais alterações fisiológicas ocorridas em pacientes infectados pelo SARS-coV2 e suas características. As alterações foram relatadas em indivíduos com e sem comorbidades.

COMPONENTE CLÍNICO INVESTIGADO
ALTERAÇÕES FISIOLÓGICAS MAIS FREQUENTEMENTE RELATADAS EM INDIVÍDUOS* COM COVID-19
Mediadores de inflamação

Metabolismo de carboidratos

Atividade cardíaca

Atividade hepática

Atividade renal
Aumento na produção de citocinas e quimiocina próinflamatórias.

Hiperglicemia; valor elevado de hemoglobina glicada; aumento na expressão da lactato desidrogenase (LDH).

Elevação da troponina cardíaca; lesão e espessamento nas cavidades cardíacas; fragmentação das placas ateroscleróticas.

Aumento na concentração sérica de alanina aminotransferase (ALT), aspartato aminotransferase (AST), bilirrubina e albumina.

Elevação da creatinina sérica e ureia; proteinúria e hematúria.

\footnotetext{
*Foram considerados indivíduos inclusos e os não inclusos nos grupos de risco para a infecção.
}

\section{Discussão}

Apesar do amplo repertório de manifestações clínicas, os casos graves da COVID19 são essencialmente caracterizados pela ocorrência de uma resposta inflamatória aguda, geralmente associada ao mau prognóstico da doença (ANTONIO et al., 2020). A inflamação é uma resposta sistêmica elaborada pelo organismo no intuito de favorecer e agilizar a eliminação de agentes nocivos. Porém, a exacerbação ou persistência da resposta inflamatória pode causar danos irreversíveis aos tecidos 
atingidos, como é possível verificar na caracterização de casos da doença (KOSUGI et al., 2020).

Registra-se que nas infecções por SARS-coV-1 (Severe Acute Respiratory Syndrome coronavírus 1) e MERS-coV (Middle East respiratory syndrome) ocorre demasiada produção de mediadores pró-inflamatórios (cascata de citocinas) no sistema pulmonar dos infectados (CHANNAPPANAVAR \& PERLMA, 2017). Na atual pandemia causada pela infecção por SARS-coV-2 (Severe Acute Respiratory Syndrome coronavírus 2) também se observa presença consistente de mediadores pró-inflamatórios: citocinas (interferon e a interleucina 6 (IL-6), especialmente) e as quimiocinas nas amostras obtidas de pacientes em estado grave da doença (ANTONIO et al., 2020).

Interferon são mediadores centrais na modulação na defesa inata contra infecção virais, o que explica o aumento em sua concentração nas amostras clínicas analisadas pelos estudos. As IL-6 atuam em diferentes etapas da infecção pelo SARS-coV-2, mas seu papel crucial é exercido na modulação da inflamação aguda registradas em pacientes graves. As quimiocinas são fortes quimiotáticos capazes de recrutar eficientemente as células e moléculas do sistema imunológico (COPERCHINIA et al., 2020). Dentro de condições homeostáticas as funções desempenhadas pelas citocinas e quimiocinas são imprescindíveis para o organismo. Todavia, o que observa nos estudos realizados é que a atuação exacerbada desses mediadores apresenta relação com a perda da atividade respiratória nos infectados pelo SARS-coV-2 (ARAÚJO-FILHO et al., 2020; CHATE et al., 2020; PAN et al., 2020).

Dados clínicos relacionam a ocorrência de diabetes com altas taxas de morbimortalidade em pacientes com COVID-19 e ao maior índice de internações prolongadas nas Unidades de Terapia Intensiva (UTI), devido as complicações metabólicas decorrentes de falhas no metabolismo dos carboidratos; bem como pela possibilidade de comprometimento na função pancreática (responsável pela secreção da insulina) e posterior desregulação na glicemia (COETZEE et al., 2020; PAPADOKOSTAKIA, TENTOLOURISB \& LIBEROPOULOSC, 2020).

A hiperglicemia inerente ao quadro de diabetes mal controlada tem associação com falhas na proliferação de macrófagos e impacta negativamente na atividade de 
neutrófilos, reduzindo a defesa inata nesses pacientes. Adicionalmente, achados clínicos evidenciaram que a hiperglicemia contribui para o desenvolvimento viral no trato respiratório assim como em diferentes órgãos do organismo humano (COETZEE et al., 2020). Hiperglicemia também potencializa a liberação de mediadores inflamatórios, o que no contexto da COVID-19 amplifica a "cascata de citocinas" e resulta na piora clínica do paciente (CERIELO, 2020).

Concomitantemente ao registro da hiperglicemia, foi observado aumento na produção da enzima lactato desidrogenase (LDH) e do seu produto metabólico, i.e. molécula de lactato, em momentos de piora clínica de pacientes com COVID-19 o que sugere fortemente a ocorrência de dano celular (CARVALHO et al., 2020; GUAN et al., 2020; LI et al., 2020b).

Os valores de hemoglobina glicada são utilizados na avaliação retrospectiva do metabolismo glicêmico ocorrido em até 3 meses anteriores, e os relatos clínicos a partir de amostras de pacientes com COVID-19 apontam que os valores altos de hemoglobina glicada oferecem risco aumentado para complicações da doença (WILLIAMISON et al., 2020).

Embora a COVID-19 seja primordialmente caracterizada como uma infecção do trato respiratório, a sintomatologia apresentada pelos infectados permite inferir sobre a ocorrência de outros agravos a saúde. Dados clínicos revelaram casos de pacientes de COVID-19 com alterações cardíacas, mas sem qualquer comprometimento na atividade respiratória, por exemplo (YANG \& JIN, 2020).

Pacientes portadores de doença cardiovascular (DCV) e COVID-19 são mais frequentemente internados em UTI, portanto, mais expostos aos procedimentos invasivos que proporcionam a ocorrência de Infecções Relacionadas a Assistência em Saúde. O agravamento no quadro clínico dos cardiopatas em virtude da COVID-19 aumenta em até $40 \%$ o risco de óbito, em comparação aos indivíduos sem relato de DCV (MARTINS et al., 2020).

O processo inflamatório inerente a COVID-19 pode alcançar o músculo cardíaco em indivíduos sem histórico de DCV, resultando em espessamento e lesões em suas 
cavidades, fenômeno evidenciado pelo aumento na liberação da troponina (NETO et al., 2020; ZHENG et al, 2020a). Ocorrência de lesões e a introdução do coronavírus nas células miocárdicas reduzem a expressão da enzima conversora da angiotensina 2 (ECA2), responsável pela conversão da angiotensina 1-7 (Ang1-7) a partir da angiotensina 2 (Ang II), que por sua vez desempenha papel protetor nos sistemas cardíaco e respiratório devido as suas propriedades anti-inflamatórias e antitrombóticas. Testes laboratorial evidenciaram altos níveis de Ang II no plasma de pacientes com COVID-19 o que reforça a interferência viral na atividade da ECA2 (OUDIT et al., 2009; GUO et al., 2020).

Relatos de aterotrombose aguda em pacientes de COVID-19 estão associados a "tempestade inflamatória" resultante da liberação demasiada de mediadores inflamatórias e apoptóticos tais como: citocinas pró-inflamatórias e fator de necrose tumoral, capazes de desestabilizar placas ateroscleróticas previamente formadas no endotélio dos vasos coronários, e consequentemente na liberação de êmbolos (LIU et al., 2020; NETO et al., 2020).

$\mathrm{Na}$ infecção pelos outros coronavírus: MERS e SARS-coV 1 existe uma correlação com danos hepáticos bem estabelecida (CHAU et al., 2004). E no curso da pandemia causada pelo SARS-coV-2 já foram relatadas lesão hepáticas em pacientes de COVID19 as quais são evidenciadas pelo aumento das proteínas aspartato aminotransferase (AST) e alanina aminotransferase (ALT) utilizada como marcadores bioquímicos para casos graves da doença (ALSAAD et al., 2018; JIN et al., 2020; ZHANG et al., 2020b).

Amostras séricas de pacientes com COVID-19 internados em UTI apresentaram elevada concentração da albumina, bilirrubina e das aminotransferases reforçando a gravidade clínica e, por conseguinte, o prognóstico desfavorável para esses indivíduos (WANG et al., 2020). Os danos no fígado, responsáveis pelo aumento dos biomarcadores supracitados, estão correlacionados a múltiplos fatores, como: i) colonização viral nos hepatócitos; ii) estresse oxidativo gerado pela hipoxia nos pacientes que apresentam insuficiência respiratória ou pela congestão hepática ocasionada pela ventilação mecânica; iii) hepatoxicidade dos fármacos empregados na 
terapêutica da doença; iv) produção das células $\mathrm{T}$ intra-hepática devido a resposta imunológica frente a colonização viral nos hepatócitos, e v) dos processos de coagulação, fibrinólise e trombocitopenia (YANG et al., 2019; KUKLA et al., 2020).

Todavia, as causas das lesões hepáticas em pacientes de COVID-19 ainda não foram totalmente elucidadas por falta de dados conclusivos a respeito da real interferência dos demais agravos no tecido hepático (cirrosse por exemplo) podem gerar no organismo dos infectado pelo SARS-coV-2 (GHOLIZADEH et al., 2020; KUKLA et al., 2020).

Presença de proteínas na urina (proteinúria) atua como diagnóstico para disfunção renal, haja vista que em condições homeostáticas a filtração glomerar não viabiliza a liberação dessas biomoléculas no ultrafiltrado renal. A constatação de proteinúria, assim como hematúria (presença de sangue na urina) foram encontradas em amostras dos pacientes graves de COVID-19 (CHENG et al., 2020). A presença de proteínas e sangue na urina pode ser explicada por: $i$ ) dano celular direto pela infeção viral (XU et al., 2020); ii) lesões renais de ordem secundária aos efeitos da infecção (ZHANG et al., 2020) e iii) ocorrência de choque séptico (BELLOMO et al., 2017).

Alteração nos níveis de creatinina e ureia sérica nos pacientes de COVID-19 sugerem disfunção renal, mas ainda segue em investigação (MOITINHO et al., 2020).

Em tempo, destaca-se que outros biomarcadores de agravos a saúde são clinicamente relacionados a COVID-19, porém devido as limitações desse trabalho: $i$ ) tempo destinado a realização da atividade proposta e $i$ ) falhas na conexão com a internet durante as interações on line para discussão esse trabalho se limitou a discutir as alterações fisiológicas relatadas com maior frequência nos artigos selecionados em sua metodologia.

\section{Considerações finais}

Os graduandos de Farmácia que participaram desse estudo irão atuar em estágios nos semestres posteriores. Portanto, os conhecimentos que eles adquiriram sobre as alterações promovidas pela infecção pelo SARS-coV-2 adquiridos podem ser transmitidos, dentro do âmbito de trabalho da Atenção Farmacêutica, para os usuários assistidos pelas instituições nas quais esses estudantes atuam como estagiários, de modo a combater as chamadas "Fake News" 
a respeito da COVID-19 e alertando sobre as alterações fisiológicas que servem como diagnóstico e/ou podem caracterizar um prognóstico da doença.

\section{Referências}

ALSAAD, K.O.; HAJEER, A.H.; BALWI, M. et al. (2018). Histopathology of Middle East respiratory syndrome coronovirus (MERS-CoV) infection - clinicopathological and ultrastructural study. Histopathology. v. 72, p. 516-524. doi:10.1111/his.13379

ANTONIO, M.V.N.; IMPERADOR, C.H.L.; ESPREAFICO JUNIOR, C.R. et al. (2020). Tempestade de citocinas naCOVID-19. ULAKES Journal of Medicine. 1(EE) p. 31-40. http://revistas.unilago.edu.br/index.php/ulakes

ARAUJO-FILHO, J.A.; SAWAMURA, M.V.; COSTA, A.N. et al. (2020). COVID-19 pneumonia: what is the role of imaging in diagnosis? Jornal Brasileiro de Pneumologia. v. 46, n. 2. https://doi.org/10.36416/1806-3756/e20200114

BELlOMO, R.; KELlUM, J.A.; RONCO, C. et al. (2017). Acute kidney injury in sepsis. Intensive Care Med. v. 43, n. 6, p. 816-28. DOI: 10.1007/S00134-017-4755-7.

BRASIL. Ministério da Saúde (2020). Ministério da Saúde confirma primeiro caso de reinfecção por Covid-19. Disponível em: https://www.gov.br/saude/ptbr/assuntos/noticias/ministerio-da-saude-confirma-primeiro-caso-de-reinfeccao-por-covid-19. Acesso em 19/12/2020 as 10:00h.

BRASIL. Ministério da Saúde (2012). Comissão Nacional de Incorporação de Tecnologias no SUS, Secretaria de Ciência, Tecnologia e Insumos Estratégicos. Uso off label: erro ou necessidade? Revista da Saúde Pública. v. 46, n. 2, p. 398-9.

CAGANAZZO, T.D.O. \& CHIARI-ANDRÉO, B.G. (2020). COVID-19: cuidados farmacêuticos durante a pandemia. Revista Brasileira Multidisciplinar. v. 23, n. 1, p. 162-178. https://doi.org/10.25061/2527-2675/ReBraM/2020.v23i1.973

CALLAWAY, E. (2020). What landmark COVID vaccine results mean for the pandemic. Nature. v. 587, n. 7834, p. 337-338. doi:10.1038/d41586-020-03248-7

CARVALHO, A.C.S.; BARROS, L.S.A.; TENÓRIO, E.C.P. et al. (2020). Altered coagulations modulators compromise COVID-19 infected patients. Brazilian Journal of health Review. v. 3, n. 5, p. 11624-11644. DOI:10.34119/bjhrv3n5-021

CHANNAPPANAVAR, R. \& PERLMAN, S. (2017). Pathogenic human coronavirus infections: causes and consequences of cytokine storm and immunopathology. Semin Immunopathol. v. 39, n. 5. p. 529-39. doi:10.1007/s00281-017-0629-X 
CHATE, R.C.; FONSECA, E.K.; PASSOS, R.B. et al. (2020). Apresentação tomográfica da infecção pulmonar na COVID-19: experiência brasileira inicial. Jornal Brasileiro de Pneumologia. v. 46, n. 2, p. 1-4. https://doi.org/10.36416/1806-3756/e20200121

CHAU, T.N.; LEE, K.C.; YAO, H. et al. (2004). SARS-associated viral hepatitis caused by a novel coronavirus: Report of three cases. Hepatology. v. 39, p. 302-310. doi:10.1002/hep.20111

CHENG, Y.; LUO, R.; WANG K. (2020). Kidney disease is associated with in-hospital death of patients with COVID-19. Kidney International. v. 97, n. 5, p. 829-838. https://doi.org/10.1016/j.kint.2020.03.005

CERIELLO A. (2020). Hyperglycemia and the worse prognosis of COVID-19. Why a fast blood glucose control should be mandatory. Diabetes Research and Clinical Practice. 163:108186. doi:10doi:10.1016/j.diabres.2020.108186

COETZEE, A.; TALIJAARD, J.I.; HUGO, S.S. et al. (2020). Diabetes mellitus and COVID19: A review and management guidance for South Africa. SAMJ. v. 110, n. 8, p. 761-766. https://doi.org/10.7196/SAMJ.2020.v110i8.14881

COPERCHINIA, F.; CHIOVATOA, L.; CROCE L. et al. (2020). The cytokine storm in COVID-19: An overview of the involvement of the chemokine/chemokine-receptor system. Cytokine and Growth Factor Reviews. v. 53, p. 25-32. https://doi.org/10.1016/j.cytogfr.2020.05.003

GUAN, W.; NI, Z.; HU, Y. et al. (2020). Clinical characteristics of coronavirus disease 2019 in China. The New England Journal of Medicine. v. 382, p. 18 https://doi.org/10.1056/NEJMoa2002032

GHOLIZADEH, P.; SAFARI, R.; MAROFI, P. et al. (2020). Alteration of liver biomarkers in patients with SARS-CoV-2 (COVID-19). Journal of Inflammation Research. v. 13 p. 285-292. doi:10.2147/JIR.S257078

GUO, T.; FAN, Y.; CHEN, M. et al. (2020). Cardiovascular implications of fatal outcomes of patients with coronavirus disease 2019 (COVID-19). JAMA Cardiology. v. 5, n. 7, p. 811-818. doi:10.1001/jamacardio.2020.1017

HARAPAN, H.; ITOH, N.; YUFIKA, A et al. (2020). Coronavirus disease 2019 (COVID-19): A literature review. Journal of Infection and Public Health. v. 3, n. 5, p. 667-673. https://doi.org/10.1016/j.jiph.2020.03.019 
JIN, X.; LIAN, J.S.; HU, J.H. et al. (2020). Epidemiological, clinical and virological characteristics of 74 cases of coronavirus-infected disease 2019 (COVID-19) with gastrointestinal symptoms. Gut. v. 69, n. 6, p. 1002-1009. doi:10.1136/gutjnl-2020-320926

KOSUGI, E.M.; LAVINSIKY, J.; ROMANO, F.R. et al. (2020). Incomplete and late recovery of sudden olfactory dysfunction in COVID-19. Brazilian Journal of Otorhinolaryngology. v. 86, n. 4, p. 490-496. https://doi.org/10.1016/j.bjorl.2020.05.001

KUKLA, M.; SKONIECZNA, K.Z.; KOTFIS, K. et al. (2020). COVID-19, MERS and SARS with concomitant liver injury - systematic review of the existing literature. Journal of Clinical Medicine. v. 9, p. 1420. doi:10.3390/jcm9051420

LI, C.; ZHAOA, C.; BAO, J. et al. (2020a). Laboratory diagnosis of coronavirus disease-2019 (COVID-19). Clinica Chimica Acta. v. 510, p. 35-46. https://doi.org/10.1016/j.cca.2020.06.045

LI, L.Q.; HUANG, T.; WANG, Y.Q. et al. (2020b). COVID-19 patients' clinical characteristics, discharge rate, and fatality rate of meta-analysis. Journal. Journal of Medical Virology. v. 92, n. 6, p. 577-583. doi:10.1002/jmv.25757

LIMA, F.L.O.; GOMES, L.N.L.; SANTOS, C.S.C. et al. (2020). Diagnosis of COVID-19: importance of laboratory tests and imaging exams. Research, Society and Development, v. 9, n. 9, e259997162. http://dx.doi.org/10.33448/rsd-v9i9.7162

LIU, Y.; YANG, Y.; ZHANG, C. et al. (2020). Clinical and biochemical indexes from 2019 nCoV infected patients linked to viral loads and lung injury. Science China Life Sciences. v. 63, n. 3, p. 364-374. https://doi.org/10.1007/s11427-020-1643-8

MARTINS, J.D.N.; SARDINHA, D.M.; SILVA, R.R. et al. (2020). Implications of COVID19 on the cardiovascular system: prognosis and intercurrences. Journal of Health \& Biological Sciences. n. 8, v. 1. p. 1-9. http://dx.doi.org/10.12662/2317-3076jhbs.v8i1.3355.p1-9.2020

MINAYO, M.C.L. (2001). Pesquisa social: teoria, método e criatividade. $19^{a}$ edição. Editora Vozes, Petropólis-RJ.

MOITINHO, M.S.; BELASCO, A.G.S.; BARBOSA, D.A. et al. (2020). Lesão renal aguda pelo vírus SARS-COV-2 em pacientes com COVID-19: revisão integrativa. Revista Brasileira de Enfermagem. v. 73 (Suppl 2):1. http://dx.doi.org/10.1590/0034-7167-2020-0354 e20200354

NETO, J.A.F.; MARCONDES-BRAGA, F.G.; MOURA, L. Z. et al. (2020). Coronavirus disease 2019 and the myocardium. Arquivo Brasileiro de Cardiologia. v. 6, n. 114, , p.10511057. https://doi.org/10.36660/abc.20200373

YANG, L.; WANG, W.; WANG, X. et al. (2019). Creg in hepatocytes ameliorates liver ischemia/reperfusion injury in a TAK1-dependent manner in mice. Hepatology. n. 69, p. 294- 


\section{3. doi:10.1002/hep.30203}

YANG, C. \& JIN, Z. (2020). An acute respiratory infection runs into the most common noncommunicable epidemic - COVID-19 and cardiovascular diseases. JAMA Cardiology. v. 5, n. 7, p. 743-744. doi:10.1001/jamacardio.2020.0934

OUDIT, G.Y.; KASSIRI, Z.; JIANG, C. et al. (2009). SARS-coronavirus modulation of myocardial ACE2 expression and inflammation in patients with SARS. European Journal of Clinical. v. 39, n. 7. p. 618-25. doi: 10.1111/j.1365-2362.2009.02153.x

PAN, Y.; GUAN, H.; ZHOU, S. et al. (2020). Initial CT findings and temporal changes in patients with the novel coronavirus pneumonia (2019-nCoV): a study of 63 patients in Wuhan, China. European Radiology. v. 30, n. 6, p. 3306-9. doi:10.1007/s00330-020-06731-x

PAPADOKOSTAKI, E.; TENTOLOURIS, N. \& LIBEROPOUPOS, E. (2020). COVID-19 and diabetes: What does the clinician need to know? Primary Care Diabetes, v. 14; p. 558-563. https://doi.org/10.1016/j.pcd.2020.06.010

SILVA, L.M.C. \& ARAÚJO, J.A. (2020). Clinical and community Pharmacist's role in the COVID-19 pandemic. Research, Society and Development, v. 9, n. 7, p. 1-14, e684974856.

SOUSA, F.C.B.; SILVA, L.C.N.T; SOUSA, M.A.A. et al. (2020). Protocols used for COVID19 diagnosis. Revista da FAESF, v. 4. Número especial COVID 19, p. 35-39.

TRITANY, R.F. \& TRITANY, E.F. (2020). Rational Use of Medicines for COVID-19 in Primary Health Care. Revista Saúde em Redes. v. 6, Supl. 2. DOI:10.18310/2446$\underline{48132020 \mathrm{v} 6 \mathrm{n} 2 \text { Suplem.3205g537 }}$

XAVIER, A.R.; SILVA, J. S.; ALMEIDA, J.P. et al. (2020). COVID-19: clinical and laboratory manifestations in novel coronavirus infection. Jornal Brasileiro de Patologia e Medicina Laboratorial. v. 56: p. 1-9.

XU, D.; ZHANG, H.; GONG, H. et al. (2020). Identification of a potential mechanism of acute kidney injury during the COVID-19 outbreak: a study based on single-cell transcriptome analysis. Intensive Care Med https://link.springer.com/article/10.1007/s00134-020-06026

ZHANG, W.; ZHAO, Y.; ZHANG, F. et al. (2020a). The use of anti-inflammatory drugs in the treatment of people with severe coronavírus disease 2019 (COVID-19): the experience of clinical immunologists from China. Clinical Immunology. 214: 108393. doi:10.1016/j.clim.2020.108393

ZHANG, Y.; ZHENG, L.; LIU. et al. (2020b). Liver impairment in COVID-19 patients: A retrospective analysis of 115 cases from a single center in Wuhan city, China. Liver International. n. 40, v. 9, p. 2095-2103. doi:10.1111/liv.14455 
ZHENG, Y.Y.; MA, Y.T.; ZHANG, J.Y. et al. (2020). COVID-19 and the cardiovascular system. Nature Reviews Cardiology. v. 17, n. 5. p. 259-260. doi: 10.1038/s41569-020-0360-5

WANG, D.; HU, B.; HU, C. et al. (2020). Clinical characteristics of 138 hospitalized patients with 2019 novel coronavírus - infected pneumonia in Wuhan, China. JAMA. v. 323, n. 11, p. 1061-1069. doi:10.1001/jama.2020.1585

WHO. World Health Organization (2020). Global Situation. In.: WHO Coronavirus Disease (COVID-19) Dashboard. Disponível em: https://covid19.who.int/. Acessado em: 04/01/2021

WILLIAMSON, E.J.; WALKER, A. J.; BHASKARANM K. et al. (2020). Factors associated with COVID-19-related death using OpenSAFELY. Nature. v. 584, p. 430-436. https://doi.org/10.1038/s41586-020-2521-4

WU, D.; WU, T.; LIU, Q. et al. (2020). The SARS-CoV-2 outbreak: What we know. International Journal of Infectious Diseases. v. 94, p. 44-48. https://doi.org/10.1016/j.ijid.2020.03.004

Financiamento: Este trabalho não recebeu nenhum financiamento.

Conflitos de interesse: Todos os autores declaram não haver conflito de interesses.

Aprovação do comitê de ética: Não aplicável.

Disponibilidade dos dados de pesquisa: Todos os dados gerados ou analisados neste estudo estão incluídos no manuscrito.

\section{Contribuição dos autores:}

Idealização: CARNEIRO, RTO. Investigação/execução da pesquisa: SILVA, CR; BARRETO, ES; SANTANA, LJ; BORGES, LR.; SILVA, YR.; redação/escrita do manuscrito: CARNEIRO, RTO 